\title{
Front Matter: Volume 6814
}

, "Front Matter: Volume 6814," Proc. SPIE 6814, Computational Imaging VI, 681401 (12 May 2008); doi: 10.1117/12.796836

SPIE. Event: Electronic Imaging, 2008, San Jose, California, United States 


\section{PROCEEDINGS \\ Electronic \\ Imaging \\ Sclence and Technology}

\section{Computational Imaging VI}

Charles A. Bouman

Eric L. Miller

llya Pollak

Editors

28-29 January 2008

San Jose, California, USA

Sponsored and Published by

IS\&T-The Society for Imaging Science and Technology

SPIE

Cosponsored by

GE Healthcare (USA) 
The papers included in this volume were part of the technical conference cited on the cover and title page. Papers were selected and subject to review by the editors and conference program committee. Some conference presentations may not be available for publication. The papers published in these proceedings reflect the work and thoughts of the authors and are published herein as submitted. The publishers are not responsible for the validity of the information or for any outcomes resulting from reliance thereon.

Please use the following format to cite material from this book:

Author(s), "Title of Paper," in Computational Imaging VI, edited by Charles A. Bouman, Eric L. Miller, Ilya Pollak, Proceedings of SPIE-IS\&T Electronic Imaging, SPIE Vol. 6814, Article CID Number (2008).

ISSN 0277-786X

ISBN 9780819469861

Copublished by

SPIE

P.O. Box 10, Bellingham, Washington $98227-0010$ USA

Telephone +1 3606763290 (Pacific Time) · Fax +1 3606471445

SPIE.org

and

IS\&T-The Society for Imaging Science and Technology

7003 Kilworth Lane, Springfield, Virginia, 22151 USA

Telephone +1 7036429090 (Eastern Time) · Fax +1 7036429094

imaging.org

Copyright (C) 2008, Society of Photo-Optical Instrumentation Engineers and The Society for Imaging Science and Technology.

Copying of material in this book for internal or personal use, or for the internal or personal use of specific clients, beyond the fair use provisions granted by the U.S. Copyright Law is authorized by the publishers subject to payment of copying fees. The Transactional Reporting Service base fee for this volume is $\$ 18.00$ per article (or portion thereof), which should be paid directly to the Copyright Clearance Center (CCC), 222 Rosewood Drive, Danvers, MA 01923. Payment may also be made electronically through CCC Online at copyright.com. Other copying for republication, resale, advertising or promotion, or any form of systematic or multiple reproduction of any material in this book is prohibited except with permission in writing from the publisher. The CCC fee code is 0277-786X/08/ $\$ 18.00$.

Printed in the United States of America.

Paper Numbering: Proceedings of SPIE follow an e-First publication model, with papers published first online and then in print and on CD-ROM. Papers are published as they are submitted and meet publication criteria. A unique, consistent, permanent citation identifier (CID) number is assigned to each article at the time of the first publication. Utilization of CIDs allows articles to be fully citable as soon they are published online, and connects the same identifier to all online, print, and electronic versions of the publication. SPIE uses a six-digit CID article numbering system in which:

- The first four digits correspond to the SPIE volume number.

- The last two digits indicate publication order within the volume using a Base 36 numbering system employing both numerals and letters. These two-number sets start with 00, 01, 02, 03, 04, 05, 06, 07, 08 , $09,0 A, O B \ldots O Z$, followed by 10-1Z, 20-2Z, etc.

The CID number appears on each page of the manuscript. The complete citation is used on the first page, and an abbreviated version on subsequent pages. Numbers in the index correspond to the last two digits of the six-digit CID number. 


\section{Contents}

vii Conference Committee

ix Stationary features and cat detection (Keynote PresentationViewgraphs) [6814-21]

D. Geman, Johns Hopkins Univ. (USA); F. Fleuret, INRIA Rocquencourt (France)

\section{KEYNOTE PRESENTATION}

681402 Fast acquisition and reconstruction in imaging enabled by sampling theory (Invited Paper) [6814-01]

Y. Bresler, Univ. of Illinois at Urbana-Champaign (USA)

IMAGE RECONSTRUCTION I

681403 Regularized estimation of Stokes images from polarimetric measurements [6814-02]

J. R. Valenzuela, J. A. Fessler, Univ. of Michigan (USA)

681404 Non-homogeneous ICD optimization for targeted reconstruction of volumetric CT [6814-03]

Z. YU, Purdue Univ. (USA); J.-B. Thibault, GE Healthcare Technologies (USA); C. A. Bouman,

Purdue Univ. (USA); K. D. Sauer, Univ. of Notre Dame (USA); J. Hsieh, GE Healthcare

Technologies (USA)

\section{GEOMETRY-BASED TECHNIQUES IN IMAGE ANALYSIS}

681407 MCMC curve sampling and geometric conditional simulation [6814-06]

A. Fan, J. W. Fisher III, Massachusetts Institute of Technology (USA); J. Kane, Shell E\&P (USA);

A. S. Willsky, Massachusetts Institute of Technology (USA)

681408 Mixing geometric and radiometric features for change classification [6814-07]

A. Fournier, X. Descombes, J. Zerubia, INRIA (France)

681409 3D object recognition using fully intrinsic skeletal graphs [6814-08]

D. Aovada, H. Krim, North Carolina State Univ. (USA)

6814 OA Content based image retrieval for matching images of improvised explosive devices in which snake initialization is viewed as an inverse problem [6814-09]

S. T. Acton, A. D. Gilliam, B. Li, Univ. of Virginia (USA); A. Rossi, Platinum Solutions (USA)

\section{SEGMENTATION}

6814 OB Segmentation of digital microscopy data for the analysis of defect structures in materials using nonlinear diffusions [6814-10]

L. M. Huffman, Purdue Univ. (USA); J. Simmons, Air Force Research Lab. (USA); I. Pollak,

Purdue Univ. (USA) 
6814 OC A new approach for joint estimation of local magnitude, decay, and frequency from single-shot MRI [6814-11]

W. Tang, S. J. Reeves, Auburn Univ. (USA); D. B. Twieg, Univ. of Alabama at Birmingham (USA)

6814 OD A novel image analysis method based on Bayesian segmentation for event-related functional MRI [6814-12]

L. Huang, M. L. Comer, Purdue Univ. (USA); T. M. Talavage, Purdue Univ. (USA) and Indiana Univ. School of Medicine (USA)

6814 OE Volumetric fMRI data analysis using an iterative classification method [6814-13]

L. Liu, K. Han, Purdue Univ. (USA); T. M. Talavage, Purdue Univ. (USA) and Indiana Univ.

School of Medicine (USA)

6814 OF Rule-based fuzzy vector median filters for 3D phase contrast MRI segmentation [6814-14] K. S. Sundareswaran, Georgia Institute of Technology (USA); D. H. Frakes, 4D Imaging Inc. (USA); A. P. Yoganathan, Georgia Institute of Technology (USA)

\section{SPARSE RECOVERY AND COMPRESSED SENSING}

$68140 \mathrm{~J}$ Greedy signal recovery and uncertainty principles [6814-18]

D. Needell, R. Vershynin, Univ. of California, Davis (USA)

6814 OK Blind reconstruction of sparse images with unknown point spread function [6814-19]

K. Herrity, Univ. of Michigan (USA); R. Raich, Oregon State Univ. (USA); A. O. Hero III, Univ. of Michigan (USA)

6814 OL Results in non-iterative MAP reconstruction for optical tomography [6814-40]

G. Cao, C. A. Bouman, K. J. Webb, Purdue Univ. (USA)

IMAGE ANALYSIS I

6814 OP A generalization of non-local means via kernel regression [6814-23]

P. Chatterjee, P. Milanfar, Univ. of California, Santa Cruz (USA)

$68140 Q \quad$ Functional minimization problems in image processing [6814-24]

Y. Kim, L. A. Vese, Univ. of California, Los Angeles (USA)

6814 OR Determining canonical views of 3D object using minimum description length criterion and compressive sensing method [6814-25]

P.-F. Chen, H. Krim, North Carolina State Univ. (USA)

\section{IMAGE RECONSTRUCTION II}

$68140 \mathrm{OU}$ A least squares approach to estimating the probability distribution of unobserved data in multiphoton microscopy [6814-27]

P. Salama, Indiana University (USA) 
$68140 \mathrm{~V}$ Progress in mesh based spatio-temporal reconstruction [6814-28]

J. G. Brankov, R. Delgado Gonzalo, Y. Yang, M. Jin, M. N. Wernick, Illinois Institute of Technology (USA)

6814 OW Diode laser absorption tomography using data compression techniques [6814-29] C. Lindstrom, Air Force Research Lab. (USA); C.-J. Tam, Taitech, Inc. (USA); R. Givens, Air Force Institute of Technology (USA); D. Davis, S. Williams, Air Force Research Lab. (USA)

$68140 X$ 3D macromolecule structure reconstruction from electron micrograph by exploiting symmetry and sparsity [6814-30]

M. W. Kim, J. Choi, J. C. Ye, Korea Advanced Institute of Science and Technology (South Korea)

6814 OY Alternating minimization algorithm for quantitative differential-interference contrast (DIC) microscopy [6814-42]

J. A. O'Sullivan, Washington Univ. in St. Lovis (USA); C. Preza, The Univ. of Memphis (USA)

IMAGE ANALYSIS II

$68140 Z$ Image filter effectiveness characterization based on HVS [6814-31]

V. V. Lukin, N. N. Ponomarenko, S. S. Krivenko, National Aerospace Univ. (Ukraine);

K. O. Egiazarian, J. T. Astola, Tampere Univ. of Technology (Finland)

681410 Multimodal unbiased image matching via mutual information [6814-32]

I. Yanovsky, Univ. of California, Los Angeles (USA); P. M. Thompson, UCLA School of Medicine (USA); S. J. Osher, Univ. of California, Los Angeles (USA); A. D. Leow, UCLA School of Medicine (USA)

681411 Technology-assisted dietary assessment [6814-33]

F. Zhu, A. Mariappan, C. J. Boushey, Purdue Univ. (USA); D. Kerr, Curtin Institute of Technology (Australia); K. D. Lutes, D. S. Ebert, E. J. Delp, Purdue Univ. (USA)

681412 New methods for fMRI data processing based on locally linear embeddings [6814-34]

L. Chaillou, I. S. Yetik, M. N. Wernick, Illinois Institute of Technology (USA)

681413 Online consistency checking for AM-FM target tracks [6814-35]

N. A. Mould, C. T. Nguyen, C. M. Johnston, J. P. Havlicek, Univ. of Oklahoma (USA)

POSTER SESSION

681414 Image mosaicking based on feature points using color-invariant values [6814-36]

D.-C. Lee, O.-S. Kwon, K.-W. Ko, Kyungpook National Univ. (South Korea); H.-Y. Lee, Samsung Advanced Institute of Technology (South Korea); Y.-H. Ha, Kyungpook National Univ. (South Korea)

681415 Inverse perspective transformation for video surveillance [6814-37]

T. E. Schouten, Radboud Univ. Nijmegen (Netherlands); E. L. van den Broek, Univ. of Twente (Netherlands) 
681416 Illumination normalization for face recognition using the census transform [6814-38] J. H. Kim, J. G. Park, C. Lee, Yonsei Univ. (South Korea)

681417 Audio-video synchronization management in embedded multimedia applications [6814-41]

H.-U. Rehman, The Univ. of Texas at Austin (USA); T. Kim, Freescale Semiconductor, Inc. (USA); N. Avadhanam, Novafora Inc. (USA); S. Subramanian, Freescale Semiconductor, Inc. (USA)

Author Index 


\title{
Conference Committee
}

\author{
Symposium Chair \\ Nitin Sampat, Rochester Institute of Technology (USA) \\ Conference Chairs
}

Charles A. Bouman, Purdue University (USA)

Eric L. Miller, Tufts University (USA)

llya Pollak, Purdue University (USA)

Program Committee

Samit Basu, GE Global Research (USA)

Thomas S. Denney, Auburn University (USA)

Peter C. Doerschuk, Purdue University (USA)

Peyman Milanfar, University of California, Santa Cruz (USA)

Joseph A. O'Sullivan, Washington University in St. Lovis (USA)

Zygmunt Pizlo, Purdue University (USA)

Stanley J. Reeves, Auburn University (USA)

Yongyi Yang, Illinois Institute of Technology (USA)

Session Chairs

Keynote Presentation

Charles A. Bouman, Purdue University (USA)

Image Reconstruction I

Eric L. Miller, Tufts University (USA)

Geometry-based Techniques in Image Analysis

Mary L. Comer, Purdue University (USA)

Segmentation

Charles A. Bouman, Purdue University (USA)

Sparse Recovery and Compressed Sensing

llya Pollak, Purdue University (USA)

Image Analysis I

Paul Salama, Indiana University-Purdue University at Indianapolis (USA)

Image Reconstruction II

Edward J. Delp, Purdue University (USA)

Image Analysis II

Mireille Boutin, Purdue University (USA) 
Downloaded From: https://www.spiedigitallibrary.org/conference-proceedings-of-spie on 26 Apr 2023

Terms of Use: https://www.spiedigitallibrary.org/terms-of-use 


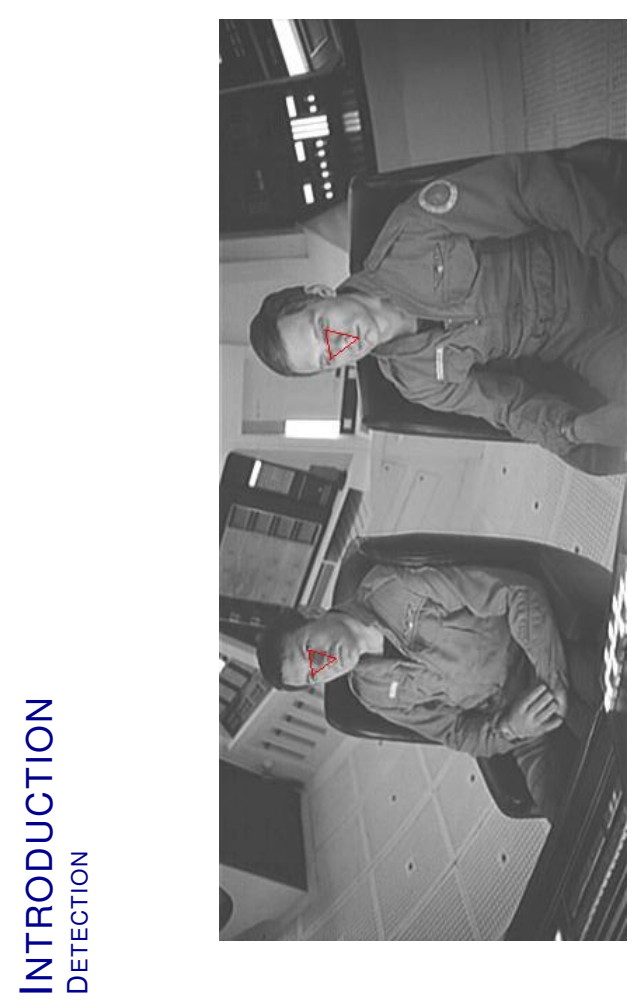

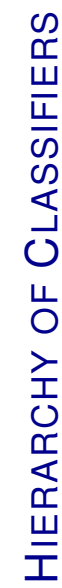
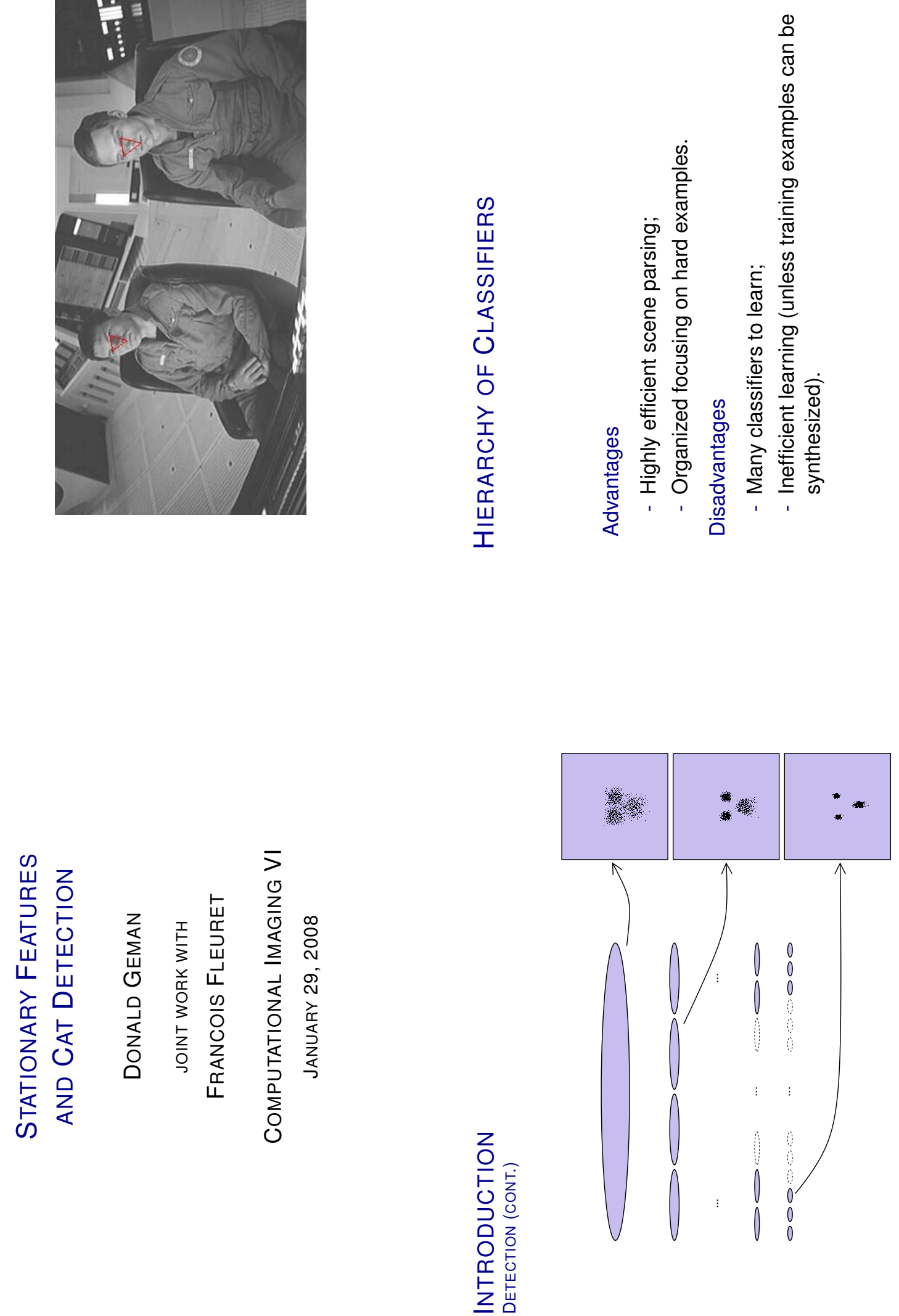

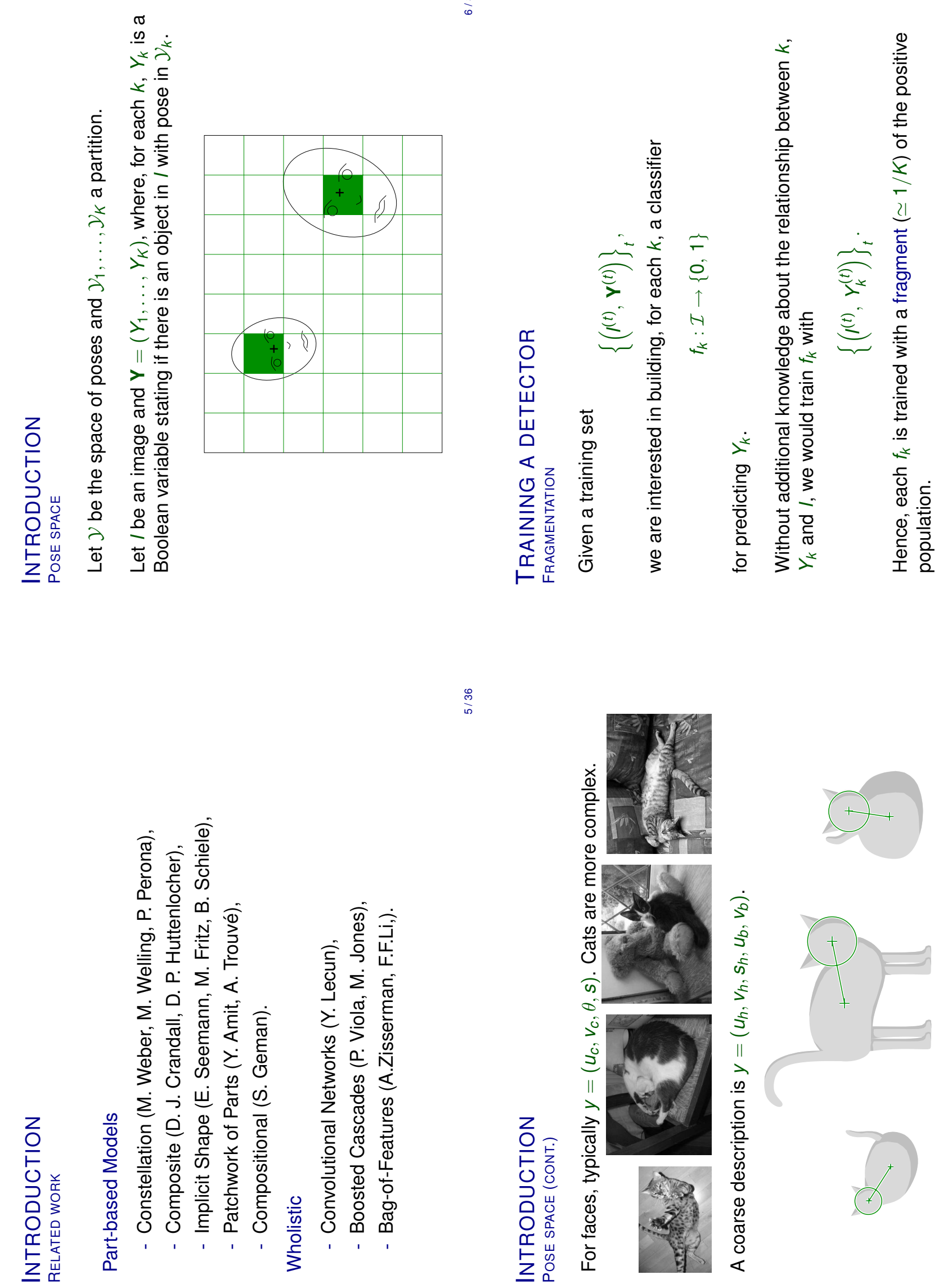

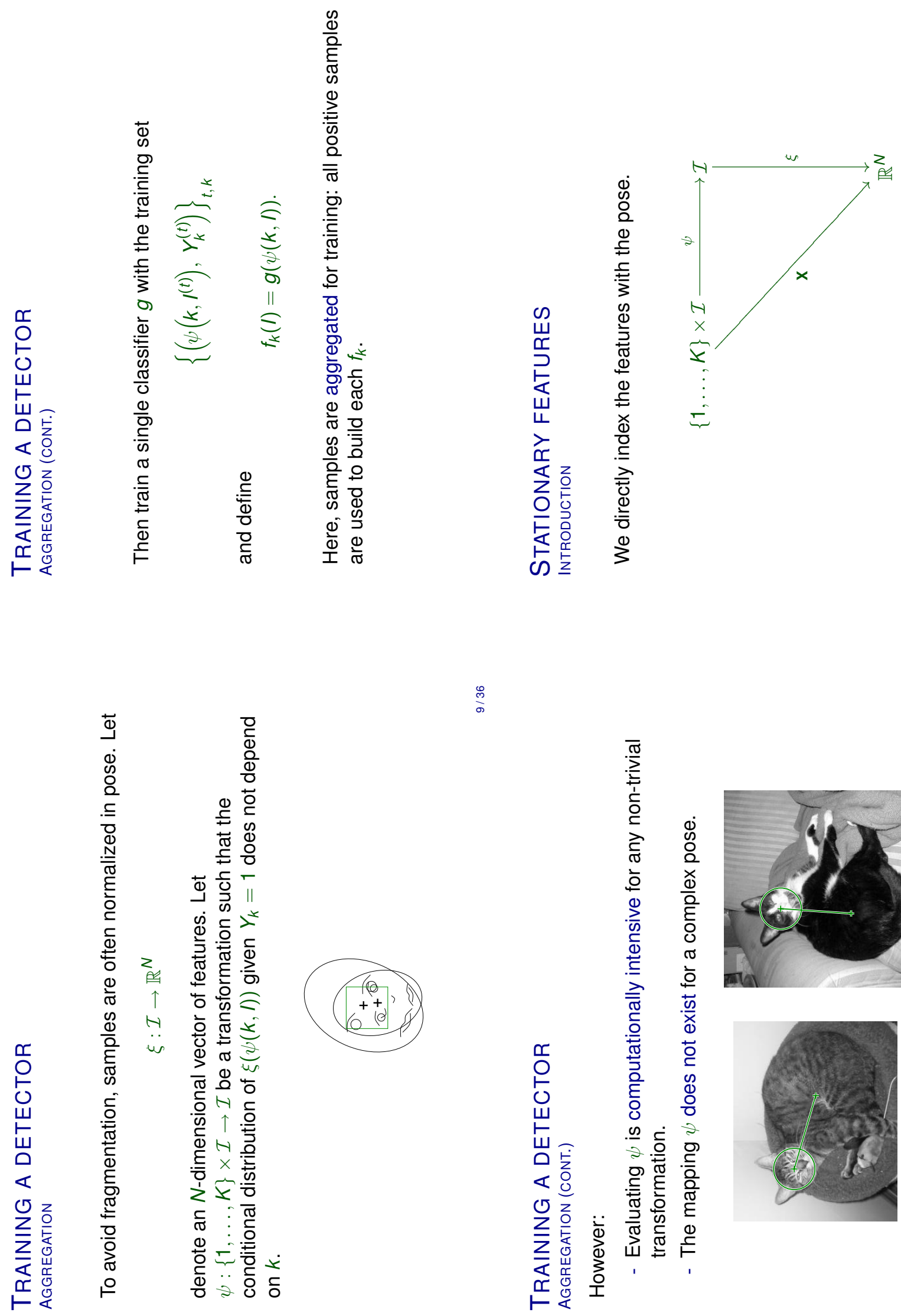

$\frac{\infty}{\infty}$

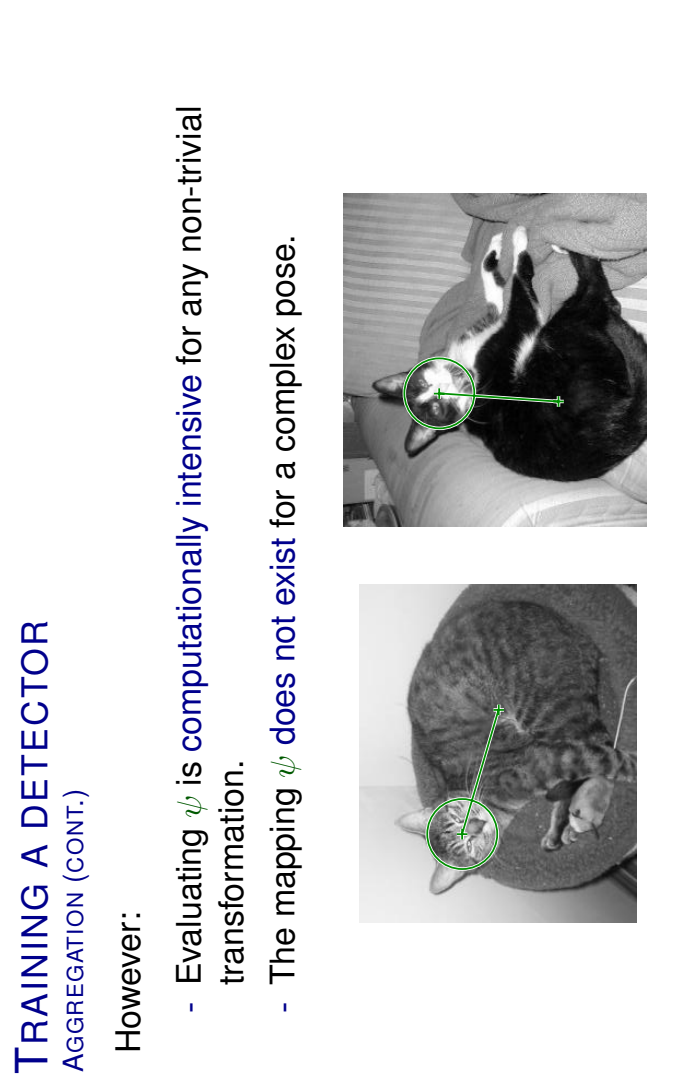

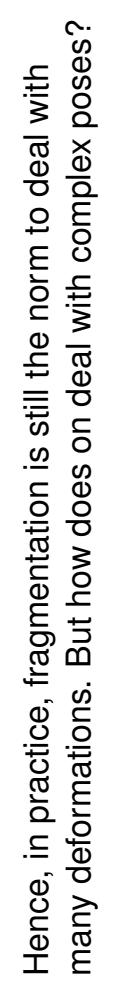



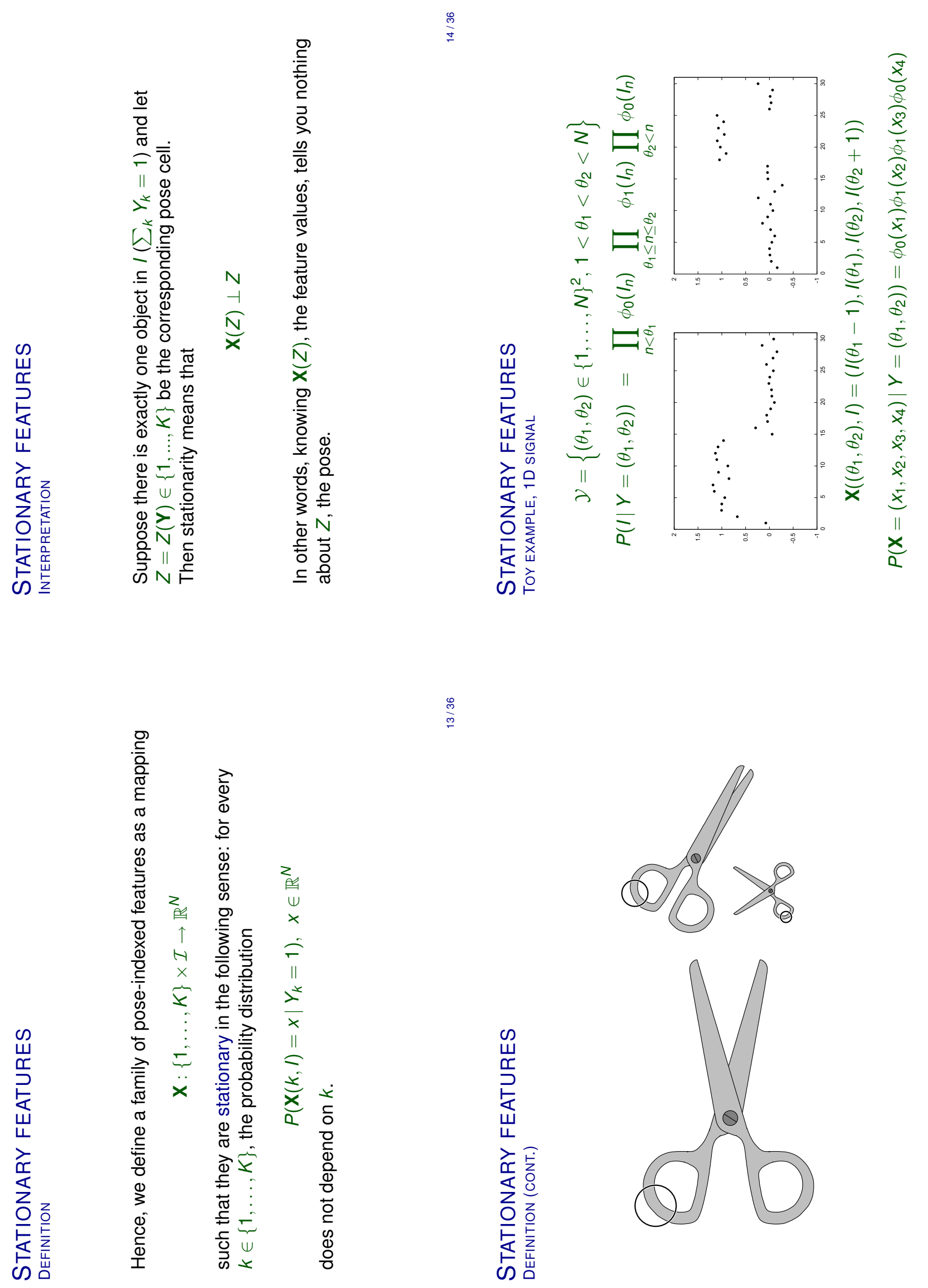

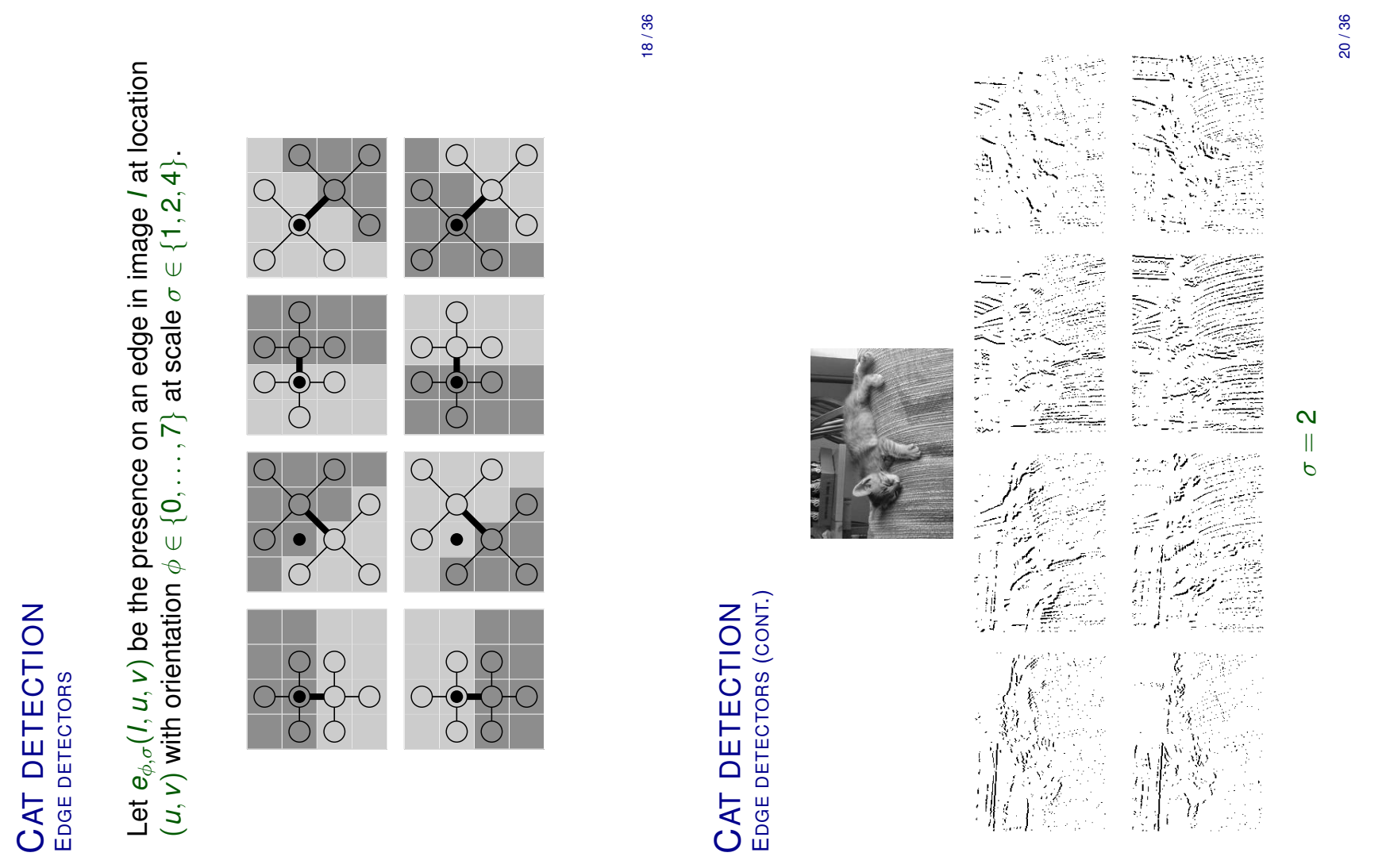

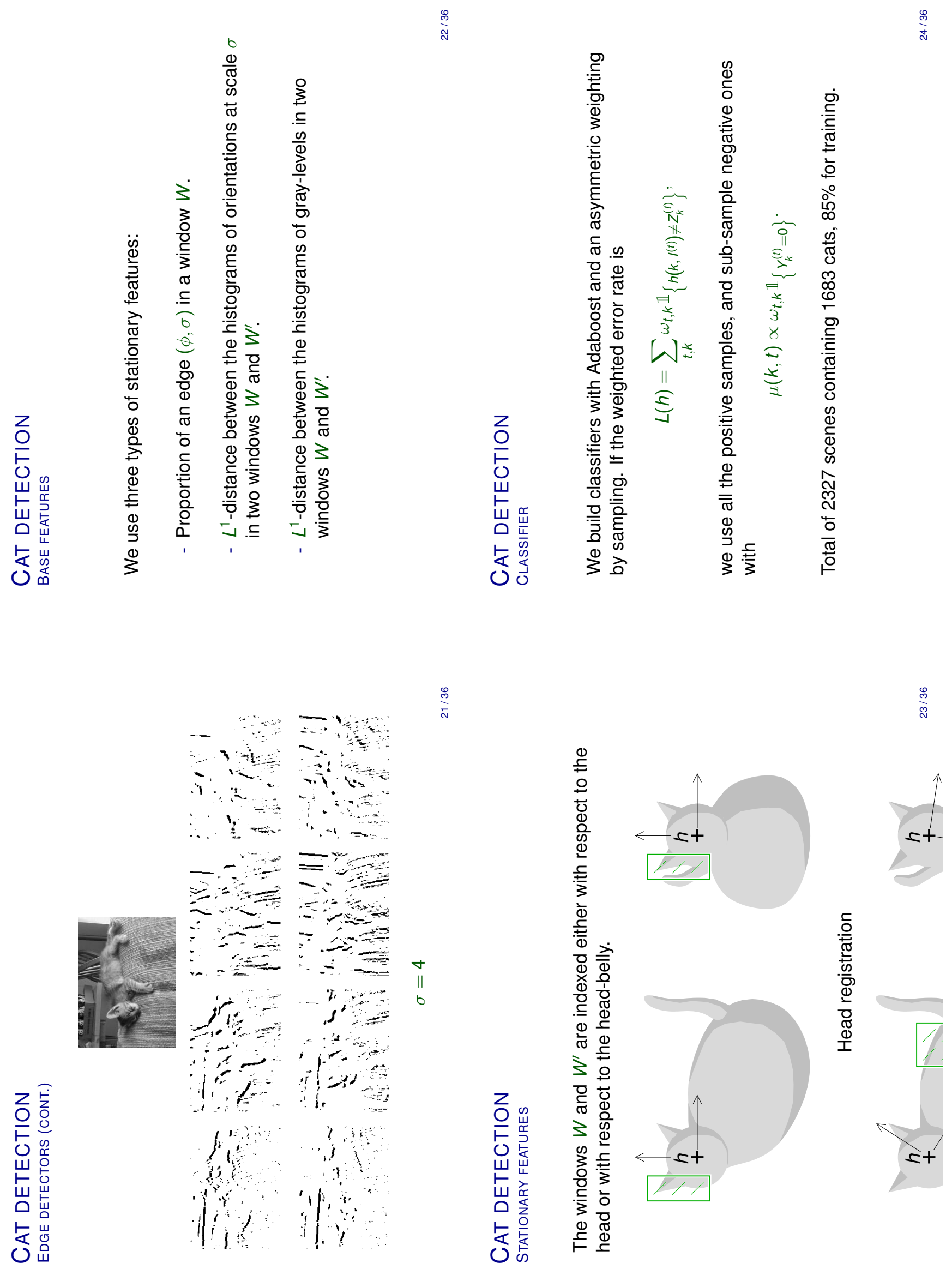

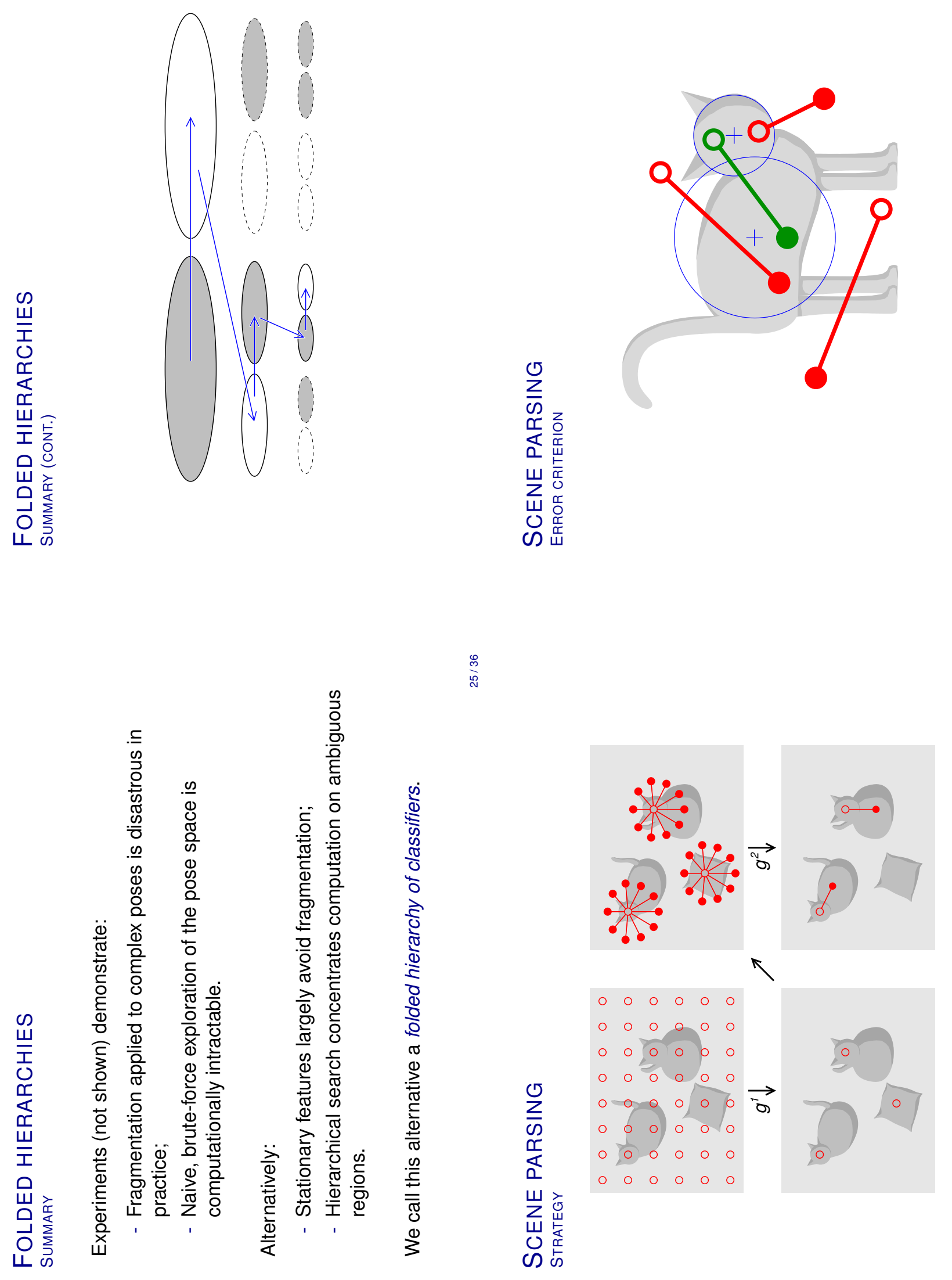

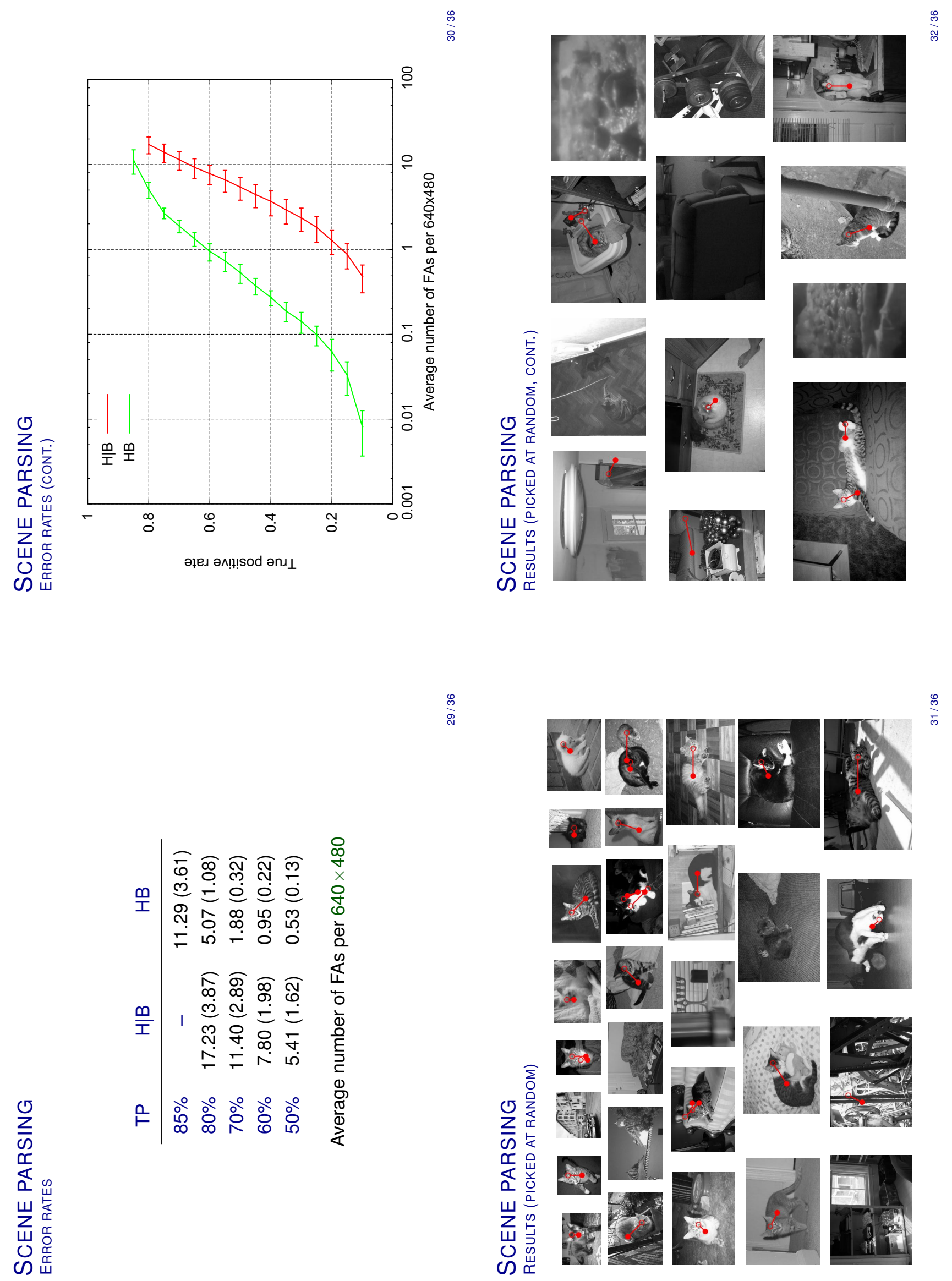

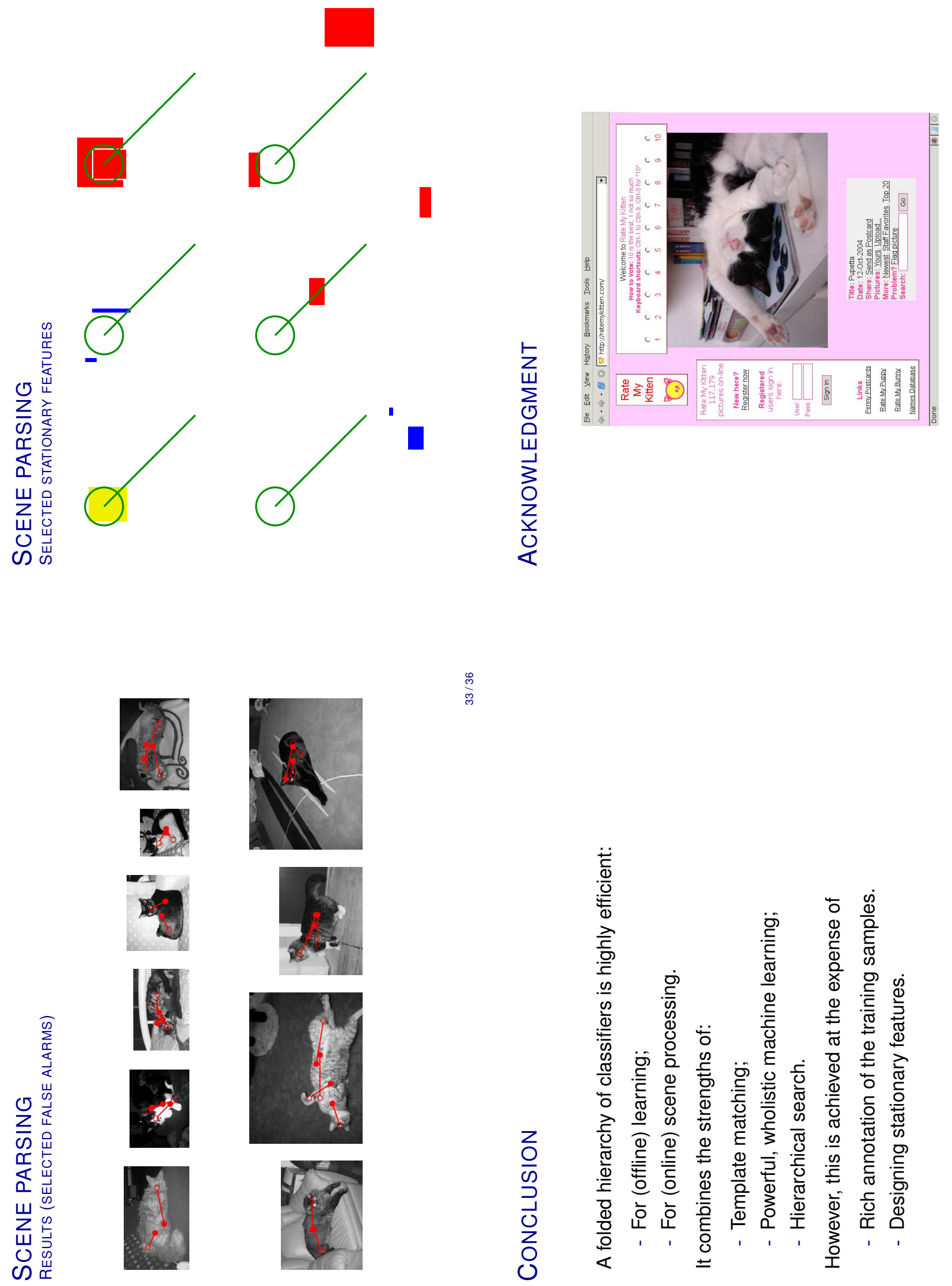
Downloaded From: https://www.spiedigitallibrary.org/conference-proceedings-of-spie on 26 Apr 2023

Terms of Use: https://www.spiedigitallibrary.org/terms-of-use 